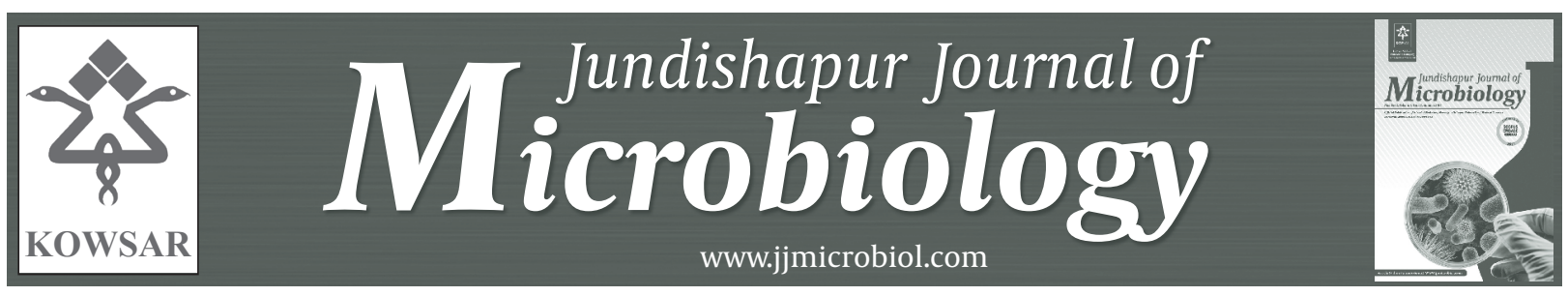

\title{
Intestinal Parasitic Infections in Chronic Psychiatric Patients in Sina Hospi- tal Shahre-Kord, Iran
}

\author{
Bahman Khalili ${ }^{1,}$, , Reza Imani ${ }^{2}$, Sanaz Boostani ${ }^{3}$ \\ ${ }^{1}$ Department of Parasitology and Mycology, Faculty of Medicine, Shahre-Kord, IR Iran \\ ${ }^{2}$ Department of Infectious Diseases, Hajar Hospital, Shahre-Kord University of Medical Sciences, Shahre-Kord, IR Iran \\ ${ }^{3}$ Department of Parasitology and Mycology, Faculty of Medicine, Shahre-Kord, IR Iran \\ *Corresponding author Bahman Khalili, Department of Parasitology and Mycology, Faculty of Medicine, Shahre-Kord, IR Iran.Tel: +98-03813334691, Fax: \\ +98-03813334911, E-mail: bahman@skums.ac.ir.
}

\section{A B S T R A C T}

Background: Although the number of infectious diseases has sharply decreased in last few decades, parasitic diseases persist in developing countries. On the other hand, chronic psychiatric patients tend to have low self-control, poor personal hygiene, long term institutionalization and extremely low self-care should be monitored for parasitic diseases since psychosocial conditions can contribute to an affinity for infectious diseases.

Objectives: The aim of study was to investigate intestinal parasites in chronic psychiatric patients.

Patients and Methods: In this cross-sectional study, all chronic psychiatric patients from Sina Hospital of Shahre-Kord University of Medical Sciences were recruited from April to November 2010. From each patient, 3 stool samples were collected every other day. Samples were transferred to Department of Parasitology of Faculty of Medicine and were examined by wet direct smear, Ziehl-Neelsen and Rayan blue trichrome stains. Direct smear was examined microscopically by performing a standard direct smear using normal saline (0.85\%) and Iodine solution (Lugol). Stools were stained by Ziehl-Neelsen and Rayan blue trichrome in order to investigate Cryptosporidium and Microsporidia respectively.

Results: Forty-seven patients (72\%) were male and 18 (28\%) were female.The minimum time of institution was 2 months and the longest period of incarceration was 152 months. The mean of hospitalization duration was 94.7 months. Forty-four cases (68\%) of participants were infected with intestinal parasites.The most frequent parasites were Blastocystis hominis in 15 cases (23\%) followed by Microsporidia in 12 cases (18.5\%), Giardia lamblia in 7 cases (11\%), Isospora in 5 cases (8\%) and Cryptosporidium in 4 cases (6.2\%), respectively.

Conclusions: Opportunistic protozoan parasites such as Microsporidia, Isospora and Cryptosporidium should be considered as a potential pathogen in this setting and more health care should be given to this specific group.

Keywords: Microsporidia; Cryptosporidium; Isospora; Psychiatric

Copyright @ (2013, Ahvaz Jundishapur University of Medical Sciences; Published by Kowsar Corp.

Article type: Research Article; Received: 08 Apr 2012, Revised: 28 Apr 2012, Accepted: 20 Jun 2012; DOI: 10.5812/jjm.5092

-Implication for health policy/practice/research/medical education:

Study can assist the Health Policy Maker as well as treatment approach in a psychiatric setting.

Please cite this paper as:

Khalili B, Imani R, Boostani S. Intestinal Parasitic Infections in Chronic Psychiatric Patients in Sina Hospital, Shahre-Kord, Iran. Jundishapur J Microbiol. 2013; 6(3):252-5. DOI:10.5812/jjm.5092

Copyright (C) 2013, Ahvaz Jundishapur University of Medical Sciences; Published by Kowsar Corp.

This is an Open Access article distributed under the terms of the Creative Commons Attribution License (http://creativecommons.org/licenses/by/3.0), which permits unrestricted use, distribution, and reproduction in any medium, provided the original work is properly cited. 


\section{Background}

Although number of infectious diseases has sharply decreased in last decades, parasitic diseases continue to maintain a presence in developing countries. Psychosocial conditions affect transmission of infectious diseases and chronic psychiatric patients due tolow self-control, poor personal hygiene, a long period of institutionalization and chronically low self-care are susceptible to parasitic diseases.

Cryptosporidium as a protozoan pathogen was first described in1976. A diarrheal illness of the parasite (Cryptosporidiosis) is typically an acute short-term infection but can become severe and non-resolving in children and immune-compromised individuals. Cryptosporidium is capable of completing its life cycle within a single host, resulting in oocyst stages which are excreted in feces that are infectious if transmitted to a new host. The usual mode of transmission appears to be direct fecal-oral spread. Contact with animals and the ingestion of contaminated water are other important routes of transmission.

The increasing population of immune-compromised persons, numerous outbreaks of Crytospodiosis and Microsporidiasis and also well-developed diagnostic procedures has placed an even greater emphasis on these pathogens. Diagnosis of Cryptosporidiosis is now largely based on observation of the oocyst parasite and conventional method of identification is the examination of fecal smears with acid fast stain. Recently, immunological evaluationsare also available which provide excellent specificity and sensitivities for laboratory uses (1-3).

Microsporidiosis can cause infection of the intestine, lung, kidney, brain, sinuses, muscles, and eyes. Intestinal Microsporidiosis is accompanied with nausea, vomiting, chronic diarrhea, wasting of the body, mal absorption of food and plenty of stool. The most prevalent Microsporidia in humans are Enterocytozoonbieneusi, Encephalitozooncuniculi, and E. intestinalis. Observation of spores in microscopic examination samples of body fluids especially fecal samples by Rayan blue stain allows for rapid diagnosis, although the exact species of Microsporidia may not be identified $(2,4,5)$.

Mental disorders are generally defined by a combination of how a person feels, acts, think or perceives. This may be associated with particular regions or functions of the brain or the rest of the nervous system. The recognition of mental health conditions has changed over time and across cultures, and there are still variations in definition, assessment and classification. Schizophrenia is a chronic, severe, and disabling brain disorder that has affected people throughout history. An estimated about one percent of world's population involved. Schizophrenia has diversetypes including paranoid, hebephrenic, catatonic, and residual schizophrenia and undifferentiated disorder (6-8).

\section{Objectives}

This cross-sectional study was carried out to find intestinal parasitic infections in chronic psychiatric patients in Sina Hospital of Shahre-Kord University of Medical Sciences.

\section{Patients and Methods}

All patients had been admitted to Sina Hospital were enrolled from April to November 2010 except for those who did not agree to participate or could not provide 3 stool samples. Data were collected by a standard questionnaire including demographical characteristics and history of the patient's disease. Physician and staff nurses provided data about each resident using standardized questionnaires. In total, 65 patients were enrolled and from each patient 3 stool samples were collected in 3 every other day. Samples were transferred to Department of Parasitology of Faculty of Medicine.

All portions of stool specimens were freshly examined under microscopic examination by preparing a standard unstained wet direct smear in physiologic saline (0.85\%) and a stained mount iodine solution (Lugol) by magnification of 400 (9). Samples were stained with ZiehlNeelsen and Rayan blue trichrome in order to investigate Cryptosporidium and Microsporidia respectively by using oil immersion (100X). Data were analyzed with SPSS version 13 and Chi-square test for qualities variables.

\section{Results}

We examined the stool specimens collected in triplicate from 65 residents who were enrolled between April to November 2010. Forty-seven patients (72\%) were male and 18 (28\%) were female. The mean age and standard deviation (SD) of participants were $41.2 \pm 10.1$ years. The youngest patient was a 17 year old boy and the eldest one was 60 years old. While the shortest time of institutionalization was 2 months, the longest time spent in hospital was 152 months. The mean of institution time was 94.7 months with SD of \pm 49.2 .

Patients were classified as 29 cases (45\%) as hebephrenic, 17 (26\%) as paranoid, 14 (22\%) as residual schizophrenia, and 3 cases in addition to schizophrenia had some mental retardations. Only one patient had catatonic schizophrenia. Regarding intestinal parasitic infections; more than $2 / 3$ (67.6\%) of patients were infected with intestinal parasites. The most frequent parasites were Blastocystishominisin in 15 cases (23\%) followed by Microsporidia in 12 cases (18.5\%), Giardia lamblia in 7 cases (11\%), Isospora in 5 cases ( $8 \%$ ) and Cryptosporidium in 4 cases (6.2\%).

Two cases were infected with helminthes including Strongyloides stercoralis and Ascaris lumbricoides 1 case (1.5\%) each. The most frequent non-pathogen parasites were Endolimax nana in 12 cases (18.5\%), followed by Entamoeba coli in 9 cases (14\%) and Iodomoeba butshlii in 
one case (1.5\%). From 15 patients that were infected with Isospora, 12 cases were hebephrenic and 3 cases were catatonic. Applying statistical tests showed a relationship between B. homonis infection and type of schizophrenia and hebephrenic patients had higher risk of infection with B. homonis. However, for Cryptosporidium, there was no relationship between infection and type of schizophrenia. No association was found between infection with $M$., Isospora and Cryptosporidium infections and gender, age, number of siblings, presence of brother or sister with similar disease in the family and duration of stay at the institution.

\section{Discussion}

Psychosocial conditions increase the chance of infectious diseases in chronic psychiatric patients due to factors such as low self-control, poorer personal hygiene, length of hospital stay and ever present low self-care are more susceptible for parasitic diseases. The study showed that $68 \%$ of participants were infected with intestinal parasites; however infection with pathogenic parasites was seen in 24 cases (37\%). Considering the unique qualities of each of the individual participant, the kind of institution they were in and for how long, combined withthe marginal self-care of the study subjects, this high rate of infection is to be expected.

Opportunistic parasites such as Microsporidia, Isospora and Cryptosporidium account for more than $50 \%$ of subjects were infected with intestinal parasites, which is higher than infection rate of the normal population and in other hospital-based studies(10-12). Given that there are no other similar studies that have focused on Misrosporidia and Isospora in this context, it is too difficult to compare and interpret findings of current study. The frequency of Cryptosporidium in the survey (6.2\%) is more likely similar to the prevalence of the parasite in hospitalized young (under 5 year old) children in Shahre-Kord as reported by Khalili from Shahre-Kord in 2006.

Studies from Isfahan and Uremia have reported a similar infection rate of Cryptosporidium in renal transplant subject, hemodialysis patients and hospital based surveys (10-12). However in contrast to our findings, many other studies have documented a higher or lower infection rate of Cryptosporidiosis in hospital-based surveys (13-17). This study has not found an association between infections with Microsporidia, Cryptosporidium and Isospo$\mathrm{ra}$ and age of patients or length of time within an institution. Many studies have reported higher Cryptosporidium infection in children and immunocompromised subjects $(18,19)$ however due to different study design, and the very few surveys with a similar setting, it is not possible to accurately compare and interpret the results.

Based on knowledge as documented in parasitologist texts, Cryptosporidiosis is an age and immune system status dependent disease. Since the participants of cur- rent study were neither children nor immunocompromised cases, it is difficult to interpret the results. In the current study, an association was found betweenBlastocystosis and schizophrenia type with higher frequency of $B$. hominis in hebephrenic patients. However, due to few positive cases and the small sample size, more studies are requiresto back up the contention that being hebephrenic is a risk factor for Blastocystosis.

\section{Acknowledgements}

This study was supported financially by Shahre-Kord University of Medical Sciences. Authors also would like to thank Dr Nickfarjam and nurses in Sina Hospital for their valuable assistance.

\section{Financial disclosure}

None declared

\section{Funding support}

None declared

\section{Authors' Contribution}

None declared

\section{References}

1. Garcia LS, Current WL. Cryptosporidiosis. Clin. Microbial Rev. 1991;4:325-358

2. Hojlyng N, Holten-Andersen W, Jepsen S. Cryptosporidiosis: a case of airborne transmission. Lancet. 1987;2(8553):271-2

3. Markel EK, Voge M, John DT. Medical parasitology. 2001.

4. Deplazes P, Mathis A, Weber R. Epidemiology and zoonotic aspects of Microsporidia of mammals and birds. Contrib Microbiol. 2000;6:236-60

5. Weber R, Deplazes P, Schwartz D. Diagnosis and clinical aspects of human microsporidiosis. Contrib Microbiol. 2000;6:166-92

6. Cross-national comparisons of the prevalences and correlates of mental disorders. WHO International Consortium in Psychiatric Epidemiology. Bull World Health Organ. 2000;78(4):413-26

7. Schizophrenia" Concise Medical Dictionary. 2010.

8. Berrios GE. Classifications in psychiatry: a conceptual history. Aust N Z J Psychiatry. 1999;33(2):145-60

9. Melvin DM, Brooke MM. Laboratory procedures for the diagnosis of intestinal parasites.

10. Khalili B, Shahabi GA, Khalili M. Prevalence of Cryptosporidium among OPD and hospitalized under 5 year's children (Shahrekord, Iran). Abstract book of 5th Iranian Congress of Parasitology and Parasitic Diseases.Tehran, Iran;: 1384. p. 94 (Full text in Persian)

11. Pestechian N, Seyrafian S, Yousefi H, Karkerdi M. Comparison of Cryptosporidium infection in hemodialysis and non hemodialysis patients (Isfahan, 1379-1380). Abstract book of 4th Iranian Congress of Parasitology and Parasitic Diseases.Mashahad, Iran:1382. p. 126 (Full text in Persian)

12. Seyrafian S, Pestehchian N, Kerdegari M, Yousefi HA, Bastani B. Prevalence rate of Cryptosporidium infection in hemodialysis patients in Iran. Hemodial Int. 2006;10(4):375-9

13. Dabirzadeh M, Bagaei M, Bokaian M, Gudarzi MR. Prevalence of Cryptosporidium in under 5 year's hospitalized children due to diarrhea (Zahedan, Iran). Journal of Gorgan University of Medical Sciences. 1382;2(11):56-61 (Full text in Persian).

14. Fakhar M, Sharif M. A study on Cryptosporidium and Giardia in- 
fection in children attended to Kerman Hospital (Kerman,1370). Abstract book of 4th Iranian Congress of Parasitology and Parasitic Diseases.Mashhad, Iran:1382. p. 127(Full text in Persian)

15. Hamedi Y, Safa O, Haidari M. Cryptosporidium infection in diarrheic children in southeastern Iran. Pediatr Infect Dis J. 2005;24(1):86-8

16. HazratiTappeh $\mathrm{KH}$, Gharavi MJ, Makhdoumi K, Rahbar M, Taghizadeh A. Prevalence of Cryptosporidium spp. Infection in Renal Transplant and Hemodialysis Patients. Iran J Public Health. 2006;35(3):54-57
17. Palit A, Sur D, MitraDhar K, Saha MR. Asymptomatic cryptosporiosis in a periurban slum setting in Kolkata, India--a pilot study. Ipn J Infect Dis. 2005;58(2):110-1

18. Hamzavim YA. survey on Cryptosporidiosis in children under 12 years old attend to Shahid Fahmideh Hospital (Kermanshah, 1374-1374). J Kerman shah Univ Med sci.1379;4(3):35-39 (Full text in Persian)

19. Lee Jk, Song HJ, Yu JR. 2005. J Parasitol. Prevalance of diarrhea caused by Criptosporidium parvum in non,HIV patient in Geollanam,do Korea. Korean;43(3):111-141 